\title{
The Effects of Cupping Therapy on Skin's Biomechanical Properties in Wistar Rats
}

\author{
Mohammad Mohsen Roostayi1, Taraneh Norouzali ${ }^{*}$, Farideh Dehghan Manshadi ${ }^{1}$, \\ Mehdi Abbasi², Alireza Akbarzadeh Baghban³ \\ ${ }^{1}$ Physiotherapy Research Center, School of Rrhabilitation, Shahid Beheshti University of Medical Sciences, \\ Tehran, Iran \\ ${ }^{2}$ Department of Anatomy, Tehran University of Medical Sciences, Tehran, Iran \\ ${ }^{3}$ Department of Biostatics, Shahid Beheshti University of Medical Sciences, Tehran, Iran \\ Email: roosta@sbmu.ac.ir, "taraneh_ph83@hotmail.com, farideh4351@gmail.com, \\ abbasima@sina.tums.ac.ir, akbarzad@gmail.com
}

Received 1 January 2016; accepted 20 March 2016; published 23 March 2016

Copyright (C) 2016 by authors and Scientific Research Publishing Inc.

This work is licensed under the Creative Commons Attribution International License (CC BY). http://creativecommons.org/licenses/by/4.0/

(c) (i) Open Access

\begin{abstract}
Cupping therapy has been widely used for clinical treatment of soft tissue lesions. The current study investigated the effects of cupping therapy on biomechanical properties of the skin in Wistar rats. 20 rats were divided into two groups: 10 in experimental and 10 in control group. Either the right or the left lower quadrants of the lumbar regions in the experimental group underwent 10 minutes daily cupping therapy for $\mathbf{1 2}$ days. The skin stiffness and ultimate tensile strength of all the rats were measured using tensiometer. The skin stiffness and ultimate tensile strength were decreased significantly in cupping side of the experimental group as compared with the non-cupping side and the control group. There were no significant differences between the non-cupping side of the experimental group and the control group. In conclusion, cupping therapy can be useful as a treatment method to reduce the skin stiffness and ultimate tensile strength.
\end{abstract}

\section{Keywords}

Cupping Therapy, Stiffness, Ultimate Tensile Strength, Wistar Rat, Skin

\section{Introduction}

In most Asian countries, Central Europe and parts of Africa, cupping therapy has been used as a non-invasive physical therapy to relieve pain and reduce the symptoms of many diseases [1] [2]. Cupping therapy is the ap-

${ }^{*}$ Corresponding author.

How to cite this paper: Roostayi, M.M., Norouzali, T., Manshadi, F.D., Abbasi, M. and Baghban, A.A. (2016) The Effects of Cupping Therapy on Skin's Biomechanical Properties in Wistar Rats. Chinese Medicine, 7, 25-30.

http://dx.doi.org/10.4236/cm.2016.71004 
plication of vacuum in one or more bell-shaped cups by means of heat or suction on different parts of the body or specific areas (meridians or acupuncture points) of the skin [3]. In many studies, a variety of effects of cupping therapy have been reported [3] [4]. Cupping therapy results in compressive stress of the soft tissue under the rim of the cup and the soft tissue enclosed by the cup is in tensile stress. Such stress may result in displacement in the layers of the cutaneous tissue and at the interface between the fat and muscle layers [5]. Thus, the amount of negative pressure and the area under the cup (diameter of the cup) may significantly affect the displacement of the layers and the interface. Certain research studies indicate that in cupping therapy, selecting the cup in appropriate diameter, applying sufficient negative pressure within the cup and considering the duration of cupping therapy are the important factors affecting the outcome of the treatment measure [5]-[11].

The diameter is regarded as an important factor in cupping therapy. Tham et al. studied the effects of cupping therapy on different skin layers from biomechanical perspective using the cups in different diameters (35, 50 and $65 \mathrm{~mm}$ ). The results of Tham et al. study indicated that increasing the aperture diameter on the suction device led to larger stresses at different tissue layers leading to larger tissue displacements and uplifts. Also, according to Tham et al. study, the cup should be as large as the anatomical location for which treatment is sought and the cups with rounded rim and diameters in the range of about $38 \mathrm{~mm}$ to $50.8 \mathrm{~mm}$ are commonly used in cupping therapy [5]. Hendriks study also demonstrated that as the aperture diameter of the cup decreased, the effect of the suction on the papillary dermis layer (the superficial layer) increased. However, as the aperture diameter of the cup increased, both papillary and reticular dermis (the deeper layer) layers were drawn into the cup [11].

The vacuum pressure is another issue that has been discussed in various studies [5]-[11]. According to Tham et al. study, increasing the vacuum pressure can increase the negative pressure in a bulb-shaped region under the centre of the cup, increasing the tensile stress and pulling the deeper layers into the cup [5]. The results of Tham et al. study were in agreement with those of Hendriks study, in that, Hendriks found that decreasing the vacuum pressure could bring only the superior soft layer (superficial tissue) into the cup, so displacement of the deeper layers was less [11].

The duration of cupping therapy is another factor warranting consideration. The results of the study examining the effects of external forces on the skin show that the external forces with low or medium duration are more appropriate for the skin. However, injuries secondary to external forces with low or medium duration may happen, but it is reversible [12]. The duration of cupping therapy has been reported to be typically between 5 - 20 minutes. However, most studies have maintained the cup on the skin for an average of 10 minutes [5] [6].

Stiffness and ultimate tensile strength are biomechanical properties of the skin. Stiffness is the rigidity of tissue against the external forces measured by modulus of elasticity and measuring linear slope of the elastic part of the stress-strain curve. If the slope of the stress-strain curve is gradual and the modulus of elasticity is low, the stiffness also will be low and vice versa [13] [14]. Ultimate tensile strength is the maximum strength of tissue against the tensile stress that is the highest point of the stress-strain curve [15] [16]. Schneider et al. examined the biomechanical changes of expanded skin conducted on pigs and found out that tissue expanders could reduce the skin stiffness and tensile strength [17]. It has been reported that the histological and histomorphological changes due to the tensile stress can change the biomechanical properties of the skin [18] [19].

The cupping therapy results in mobilizing and stretching of the skin and deeper soft tissue by pulling them into the cup and away from underlying structures [20] [21]. However, what happens to the underlying structures in terms of the biomechanical properties of the skin is not well known and most studies examined the effects of cupping therapy on relieving the symptoms of diseases [1]-[22] rather than its biomechanical effects. The purpose of the present study was to examine the effects of cupping therapy on the skin stiffness and ultimate tensile strength in Wistar rats.

\section{Materials and Methods}

An experimental study was designed in that 20 healthy male Wistar rats, eight weeks old and with a mean weight of 300 to $350 \mathrm{~g}$ were selected by the conveniencenon-probability sampling method and were randomly assigned into two equal groups: experimental and control. All the rats were obtained from the Department of Anatomy, Tehran University of Medical Sciences, Tehran, Iran. The rats were maintained under controlled temperature of $25^{\circ} \mathrm{C} \pm 3^{\circ} \mathrm{C}$ in $12 \mathrm{~h}$ light $/ 12 \mathrm{~h}$ darkness schedule. Food and tap water were freely available in the cages. The study was approved by the Institutional Medical Ethics Committee of Tehran University. The rats who developed any diseases or skin injuries were excluded from the study.

The equipment for cupping therapy included a plastic cup, a hand-pump to create negative pressure and a 
gauge to control the amount of negative pressure. The gauge was calibrated in the center of control (Yeka Instrumentation Engineering Company) to ensure the reliability and accuracy of the measurement. The results were compared with the Mc-Leod gauge.

In the experimental group, five rats randomly received cupping on the right lower quadrant of the dorsal region and the other five on the left. So, the contralateral side of the cupping receiving side was considered as non-cupping receiving side in the experimental group. The aim of selecting the control group was comparing the skin stiffness and ultimate tensile strength in the cupping receiving and non-cupping receiving sides of the experimental group with the skin stiffness and ultimate tensile strength in the control group.

The rats were prepared for the study by an animal expert to keep them in right position and a clean green cloth was put on their eyes to relax them. The hair of the cupping receiving side was cut carefully every three days by haircut machine (Mozer made in Germany) due to natural growth of the hair. Then, an ultrasonic gel was rubbed on the rim of the cup to increase the interface between the cup and the skin and not letting the air going into the up. According to Tham et al. recommendation [5], the cup diameter was selected based on the extent of cupping receiving side $(\mathrm{D}=4 \mathrm{~cm}$ ) and was placed perpendicular on the involved area. A pilot study was conducted on four rats to find out the tolerable negative pressure with no ecchymosis or skin damage which was discovered to be $20 \mathrm{mmHg}$. Thus, the negative pressure created up to $20 \mathrm{mmHg}$ to take the skin enough into the cup and the pressure was kept equal in all the sessions of cupping therapy. So, the cupping receiving side of the experimental group was cupped for 10 min over 12 consecutive sessions. On day 12, the hair of the rats in the non-cupping receiving side of the experimental group and control group was cut completely by the haircut machine. Of the 10 rats in the control group, five rats were randomly selected for skin sampling from the right lower quadrant and the remaining rats for left lower quadrant. Then, the rats were languished by ether in a closed container and were anesthetized using the insulin syringe of $0.2 \mathrm{cc}$ per $100 \mathrm{~g}$ of body weight intra-peritoneal injection of a 9:1 ratio mixture solution of ketamine hydrochloride $10 \%$ (100 mg/kg; Imalgène $1000^{\circledR}$, Rhône Mérieux, Lyon, France) andxylazine 2\% (20 mg/kg; Sigma Chemical Co., Madrid, Spain). The skin of the rats in all groups was marked by a marker and a ruler in the length of $7 \mathrm{~cm}$ and width of $2 \mathrm{~cm}$ in axial direction, so that the cupping receiving side was in the middle. The marked skin samples were separated from the body using scissors and clamp and the lumpy edges of the skin samples were cut by scalpel smoothly and regularly. The skin samples were placed in separate labeled petri dishes containing normal saline 0\%/9\% and were taken to Department of Physical Therapy, Biomechanical Research Laboratory, Tarbiat Modares University, Tehran, Iran in less than 3 h. The biomechanical parameters of the skin such as stiffness and ultimate tensile strength were assessed with a tensiometer (Zwick/Roell 2/5, Germany) that its calibration had been confirmed already. At the end of the skin sampling procedure, all the anesthetized rats were sacrified by atlanto-occipital dislocation and were delivered to the Department of Anatomy, Tehran University of Medical Sciences, Tehran, Iran. Tensiometery was carried out by an experienced personnel in Biomechanical Research Laboratory, Tarbiat Modares University, Tehran, Iran.

Dimensions of the skin samples (width, length and diameter) were measured using a digital caliper ( $\pm 0.01 \mathrm{~mm}$, Absolute Digimatic \#500-197, Mitutoyo Corp., Kawasaki, Japan) and the relevant information was recorded on the software. The skin sample was fixed in the upright position between the two clamps of the tensiometer, so that the cupped site was located in the middle. The distance between the two clamps was $3 \mathrm{~cm}$ and the ends of the skin sample were placed between two sandpapers to prevent from slipping during tension. Before the main tensile test, a little tension (preload) was applied to take the slack of the skin sample. Tensiometery was done along the longitudinal axis of the skin sample and at a speed of $20 \mathrm{~mm} / \mathrm{min}$ and the stress-strain curve was obtained.

In this study, Shapiro-wilk test was used to examine the quantitative variables and Levene's test was used to examine the equality of variances. The experimental and control groups were compared using independent sample t-test and the skin of the cupping receiving side and non-cupping receiving side in the experimental group was compared using paired sample t-test. All analyses were performed using SPSS version 18 (SPSS Inc, Chicago, IL, USA) and charting was done by Excel 2007 (Excel 2007; Microsoft Corp., Redmond, WA, USA). A p value $<0.05$ was considered to be statistically significant.

\section{Results}

In this study, the skin stiffness and ultimate tensile strength in Wistar rats were assessed and compared both between and among groups. Descriptive statistics for the skin stiffness and ultimate tensile strength are shown in Table 1 in different groups. The Shapiro-Wilk test showed that the data distribution had a normal distribution (P = $0.132)$ and the equality of variances was accepted by the Leven's test $(P=0.223)$. 
Table 1. Descriptive statistics for the skin stiffness and ultimate tensile strength in different groups.

\begin{tabular}{cccccccc}
\hline Variables & Groups & Sample size & Mean & $\begin{array}{c}\text { Standard } \\
\text { deviation }\end{array}$ & Minimum & Maximum \\
\hline Skin & Control & 10 & 4.33 & 1.01 & 3.33 & 5.36 \\
stiffness & non-cupping & 10 & 4.46 & 1.49 & 3.01 & 5.93 & 4.33 \\
& cupping & 10 & 2.98 & 1.29 & 1.63 & & \\
Ultimate tensile & & & & & & \\
strength & Control & 10 & 3.61 & 0.71 & 2.92 & 5 \\
& non-cupping & 10 & 3.80 & 1.18 & & 3.41 \\
\hline
\end{tabular}

Independent sample t-test assuming equal variances showed that the skin stiffness and ultimate tensile strength between the non-cupping receiving side in the experimental group and control group had no statistically significant differences $(\mathrm{P}=0.813, \mathrm{P}=0.668)$. Paired sample t-test was used to compare the efficacy of the cupping therapy between the cupping receiving side and non-cupping receiving side in the experimental group. It was indicated that the skin stiffness and ultimate tensile strength in the cupping receiving side of the experimental group decreased significantly $(\mathrm{P}=0.048, \mathrm{P}=0.029)$. For more analysis, independent sample t-test was used to compare the skin stiffness and ultimate tensile strength between the cupping receiving side of the experimental group and control group. The results indicated that cupping therapy decreased the skin stiffness and ultimate tensile strength in the cupping receiving side of the experimental group $(\mathrm{P}=0.018, \mathrm{P}=0.008)$. Diagram 1 and Diagram 2 present the mean skin stiffness and ultimate tensile strength in the control, non-cupping receiving and cupping receiving groups.

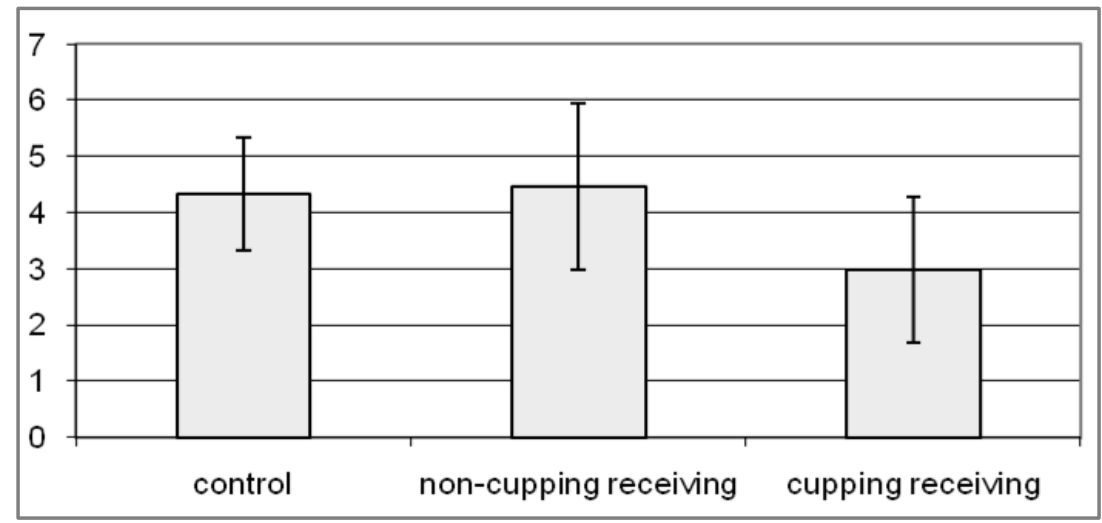

Diagram 1. Mean stiffness in the control, non-cupping receiving and cupping receiving groups.

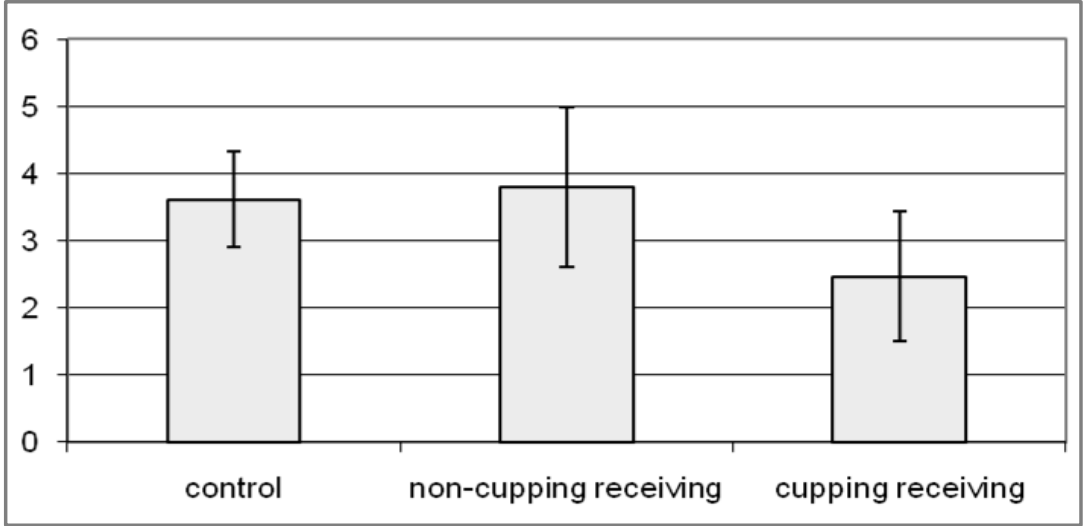

Diagram 2. Mean ultimate tensile strength in the control, non-cupping receiving and cupping receiving groups. 


\section{Discussion}

The purpose of the study was to examine the effects of cupping therapy on the skin stiffness and ultimate tensile strength in Wistar rats. According to the results of the study, cupping therapy decreased the skin stiffness and ultimate tensile strength significantly which has also been shown in Schneider et al. study [17]. While the results were the same, pulling directions of the skin were different in the two studies. In the present study, the tensile stresses pulled the skin in all directions, while in Schneider et al. study [17] the tensile stresses were in two opposite directions. It suggests that the direction of stress may have no effect on the skin stiffness and ultimate tensile strength.

In the present study, the effects of cupping therapy on biomechanical properties of the skin were studied with constant size of the cup $(\mathrm{D}=4 \mathrm{~cm})$ and constant negative pressure $(20 \mathrm{mmHg})$. The results of some studies indicated that increasing the aperture diameter on the suction device leads to larger stresses at different tissue layers, larger tissue displacements and uplifts [5]-[11]. So, it is expected that cupping therapy with the larger cup size could change the biomechanical properties of the skin in different deep layers which warrants further studies. It has also been reported that increasing the vacuum pressure results in increasing the tensile stresses in a bulb-shaped region under the center of the cup and the increased pressure draws the deeper layers into the cup [5]. Therefore, increasing the vacuum pressure during cupping therapy may result in different changes on biomechanical properties of the skin which requires further investigation.

Our findings showed that the skin stiffness decreased in cupping receiving side of the experimental group which has also been indicated in Panjabi et al. study [23]. So, it seems that cupping could lead to softening the adhesions and releasing the tissues by decreasing the skin stiffness and increasing the flexibility. Thus, we may recommend using cupping therapy for relieving scar tissue, reducing adhesions and improving immobility.

According to the results of the present study, the skin stiffness and modulus of elasticity in cupping receiving side of the experimental group decreased significantly. Our findings do not support those of Argenta et al. [19] and Xu et al. [24] studies, in that they reported an increase in the skin stiffness and ultimate tensile strength due to increased amount of collagen. One possible explanation for this discrepancy is that the kind and direction of tensile stresses are different between the current study and above studies. Another explanation may be related to the direction of pulling the skin, in that in our study, the skin was pulled in all directions but in Argenta et al. and $\mathrm{Xu}$ et al. studies, the skin was pulled in two different directions.

We recommend further studies to evaluate the effects of cupping therapy on the skin and underlying tissues from histological and histomorphological perspectives and also on different skin layers using the cups with different diameters, durations and pressures. We also recommend further investigations regarding the effects of cupping therapy on increasing the joint range of motion and the skin adhesions after injuries and surgeries. In our study, the effects of tensile stress caused by negative pressure have been evaluated just on the skin, but according to the Tham et al. study based on the effects of tensile stress on deeper tissue layers [5], it is likely that cupping can affect the limitation of the motion secondary to soft tissue stiffness.

\section{Conclusion}

We can conclude that cupping therapy may be effective in decreasing the skin stiffness and ultimate tensile strength in Wistar rats. So it may seem that this technique can be used effectively in soft tissue mobilization in some conditions, such as fascial restrictions and scar tissue.

\section{Acknowledgements}

My sincere thanks and appreciate to the students and colleagues at the Department of Anatomy, Tehran University of Medical Sciences, Tehran, Iran and Biomechanical Research Laboratory, Tarbiat Modares University, Tehran, Iran.

\section{References}

[1] Kim, J.I., Lee, M.S., Lee, D.H., Boddy, K. and Ernst, E. (2009) Cupping for Treating Pain: A Systematic Review. Evidence Based Complementary and Alternative Medicine, 2011, Article ID: 467014.

[2] Michalsen, A., Bock, S., Lüdtke, R., Rampp, T., Baecker, M., et al. (2009) Effects of Traditional Cupping Therapy in Patients with Carpal Tunnel Syndrome: A Randomized Controlled Trial. The Journal of Pain, 10, 601-608. 
http://dx.doi.org/10.1016/j.jpain.2008.12.013

[3] Bhikha, R., Manxiwa, F., Qubuda, T., Bulane, T., Kamdar, Z., et al. (2008) Pilot Research Project Conducted at the University of Western Cape Therapeutic Cupping as Adjunctive Therapy in the treatment of Diabetes. Evaluation Report.

[4] Lee, M.S., Kim, J.I. and Ernst, E. (2011) Is Cupping an Effective Treatment? An Overview of Systematic Reviews. Journal of Acupuncture and Meridian Studies, 4, 1-4. http://dx.doi.org/10.1016/S2005-2901(11)60001-0

[5] Tham, L., Lee, H. and Lu, C. (2006) Cupping: From a Biomechanical Perspective. Journal of Biomechanics, 39, 21832193. http://dx.doi.org/10.1016/j.jbiomech.2005.06.027

[6] Dayer-Berenson, L. (2010) Cultural Competencies for Nurses: Impact on Health and Illness. Jones \& Bartlett Learning, United States of America.

[7] Hecker, H., Steveling, A., Peuker, E. and Kastner, J. (2005) Practice of Acupuncture: Point Location, Treatment Options, TCM Basics. 2nd Edition, George ThiemeVerlag, Germany, 37 p.

[8] Fei, X. and Mu, J. (2000) Acupuncture and Moxibustion. 6th Edition, Science Press, Beijing, 242 p.

[9] Lee, M.S., Choi, T.Y., Shin, B.C., Han, Ch. and Ernst, E. (2010) Cupping for Stroke Rehabilitation: A Systematic Review. Journal of the Neurological Sciences, 294, 70-73. http://dx.doi.org/10.1016/j.jns.2010.03.033

[10] Lee, M.S., Choi, T.Y., Shin, B.C., Kim, J.I. and Nam, S.S. (2010) Cupping for Hypertension: A Systematic Review. Clinical and Experimental Hypertension, 32, 423-425. http://dx.doi.org/10.3109/10641961003667955

[11] Hendriks, F.M. (2005) Mechanical Behaviour of Human Epidermal and Dermal Layers in Vivo. Technische Universiteit Eindhoven, Netherlands, 2-13.

[12] Sanders, J.E., Goldstein, B.S. and Leotta, D.F. (1995) Skin Response to Mechanical Stress: Adaptation Rather than Breakdown-A Review of the Literature. Journal of Rehabilitation Research and Development, 32, 214-226.

[13] Curwin, S. (2011) Joint Structure and Function. In: Levangie. P. and Norkin, C., Eds., Joint Structure and Function, 5th Edition, F. A. Davis Company, United States of America, 81-82.

[14] Wenham, M. (2000) 200 Science Investigations for Young Students: Practical Activities for Science 5-11. Sage Publications Limited, London, 126 p.

[15] Degarmo, E., Black, J. and Kohser, R. (2003) Materials and Processes in Manufacturing. 9th Edition, John Wiley \& Sons, New York, $31 \mathrm{p}$.

[16] Smith, W.F. and Hashemi, J. (2006) Foundations of Materials Science and Engineering. 4th Edition, McGraw-Hill Higher Education, New York, 223 p.

[17] Schneider, M.S., Borkow, J.E., Cruz, I.T., Marangoni, R.D., Shaffer, J., et al. (1988) The Tensiometric Properties of Expanded Guinea Pig Skin. Plastic and Reconstructive Surgery, 81, 398-405. http://dx.doi.org/10.1097/00006534-198803000-00015

[18] Melis, P., Noorlander, M.L., van der Horst, C.M. and van Noorden, C.J. (2002) Rapid Alignment of Collagen Fibers in the Dermis of Undermined and Not Undermined Skin Stretched with a Skin-Stretching Device. Plastic and Reconstructive Surgery, 109, 674-680. http://dx.doi.org/10.1097/00006534-200202000-00038

[19] Argenta, L.C., Marks, M.W. and Pasyk, K.A. (1985) Advances in Tissue Expansion. Clinics in Plastic Surgery, 12, 159-171.

[20] Roostayi, M.M., Bagheri, H., Talebian, S., Firooznia, K., Razi, M., et al. (2009) The Effects of vacuumic Bracing System on the Patellofemoral Articulation in Patients with Patellofemoral Pain Syndrome. Complementary Therapies in Clinical Practice, 15, 29-34. http://dx.doi.org/10.1016/j.ctcp.2008.09.008

[21] Waaldyk, A. Traditional Chinese Medicine. http://www.naturaltherapypages.com.au/connect/waaldyk/

[22] Hssanien, M.M.R, Mansoura Fawaz, S., Ahmed, A.F., Al Emadi, S. and Hammoudeh, M. (2010) Effect of Cupping Therapy in Treating Chronic Headache and Chronic Back Pain at Al Heijamah Clinic HMC. World Family Medicine Journal, 8, No. 3.

[23] Panjabi, M.M. and White, A.A. (2001) Biomechanics in the Musculoskeletal System. Churchill Livingstone, Philadelphia, 123-125.

[24] Xu, F. and Lu, T. (2011) Introduction to Skin Biothermomechanics and Thermal Pain. Springer, Berlin Heidelberg, $110 \mathrm{p}$. 